\title{
EFFECTS OF RECREATIONAL SOCCER ON FAT MASS IN UNTRAINED SEDENTARY ADULTS: A SYSTEMATIC REVIEW WITH META-ANALYSIS
}

review paper

() Wroclaw University of Health and Sport Sciences

DOI: https://doi.org/10.5114/hm.2022.109797

\author{
FILIPE MANUEL CLEMENTE ${ }^{1,2}{ }^{\oplus}$, FRANCISCO TOMÁS GONZÁLEZ-FERNÁNDEZ $^{3,4 \oplus,}$ \\ HALIL IBRAHIM CEYLAN $^{5}{ }^{\circledR}$, RUI SILVA $^{1 \oplus}{ }^{\circledR}$, RODRIGO RAMIREZ-CAMPILLO $^{6 \oplus}$ \\ ${ }^{1}$ Escola Superior Desporto e Lazer, Instituto Politécnico de Viana do Castelo, Viana do Castelo, Portugal \\ ${ }^{2}$ Instituto de Telecomunicações, Delegação da Covilhã, Covilhã, Portugal \\ ${ }^{3}$ Department of Physical Activity and Sport Sciences, Comillas Pontifical University, Palma, Spain \\ ${ }^{4}$ SER (Salud, Educación y Rendimiento) Research Group, Comillas Pontifical University, Palma, Spain \\ ${ }^{5}$ Department of Physical Education and Sports Teaching, Kazim Karabekir Faculty of Education, Ataturk University, \\ Erzurum, Turkey \\ ${ }^{6}$ Department of Physical Activity Sciences, University of Los Lagos, Santiago, Chile
}

\begin{abstract}
Purpose. The aim of this systematic review with meta-analysis was to assess the effects of recreational soccer on fat mass in untrained sedentary adults.

Methods. The following databases were searched: Web of Science, Scopus, SPORTDiscus, and PubMed. The inclusion criteria were: (i) sedentary adult population not restricted to sex, clinical condition; (ii) exposure to intervention programs based on soccer; (iii) intervention groups compared with passive or active (not related with soccer) control groups; (iv) fat mass (indirectly or directly measured); (v) randomized controlled trials. The database search initially yielded 4565 titles. From those, 22 articles were eligible for the systematic review and meta-analysis. The age of the included population varied from 20 to 68 years.

Results. No significant differences in fat mass changes were found between recreational soccer groups and active controls $\left(E S=-0.070\right.$, trivial; $95 \% C I:-0.28$ to $0.14 ; p=0.505 ; I^{2}=0.0 \%$; Egger's test $\left.p=0.986\right)$. However, significant changes in fat mass were observed between recreational soccer groups and passive controls $(E S=-0.43$, small; $95 \% C I$ : -0.59 to -0.27 ; $p<0.001 ; I^{2}=35.0 \%$; Egger's test $p=0.652$ ).

Conclusions. Regular participation in recreational soccer is as effective as participation in analytical exercise approaches such as continuous running to produce significant fat mass reductions in untrained sedentary populations, independently of sex and clinical condition. However, recreational soccer arouses a greater interest as it has the advantage of increasing the motivation to maintain participation in an active lifestyle.
\end{abstract}

Key words: football, body fat, body composition, health promotion

\section{Introduction}

The deleterious effects of a sedentary lifestyle are well known, and there is extensive literature regarding the different implications that sedentary lifestyle has on different structures of the human body (e.g., skeletal muscle, bone, organs, and adipose tissues) [1, 2]. To prevent these effects, there are non-pharmacological strategies that can produce positive adaptations in the general health, body composition, and physical performance among untrained individuals [3].

One of the main consequences of sedentary lifestyle is the excessive accumulation of subcutaneous and internal fat mass in the whole human body, which, in turn, leads to associated type 2 diabetes, hypertension, and cardiovascular and respiratory diseases [4]. In fact, a higher fat mass to fat-free mass ratio (more fat mass than fat-free mass) has been related

Correspondence address: Filipe Manuel Clemente, Escola Superior Desporto e Lazer, Instituto Politécnico de Viana do Castelo, Rua Escola Industrial e Comercial de Nun’Álvares, 4900-347 Viana do Castelo, Portugal, e-mail: filipe.clemente5@gmail.com, https://orcid.org/0000-0001-9813-2842

Received: August 13, 2021

Accepted for publication: September 30, 2021

Citation: Clemente FM, González-Fernández FT, Ceylan HI, Silva R, Ramirez-Campillo R. Effects of recreational soccer on fat mass in untrained sedentary adults: a systematic review with meta-analysis. Hum Mov. 2022;23(3):15-32; doi: https:// doi.org/10.5114/hm.2022.109797. 
to sarcopenic obesity (e.g., muscle degeneration concurrent with fat accumulation) [5]. Furthermore, maintaining this type of lifestyle in long-term can cause chronic diseases that limit life quality and body functionality, which is related to higher levels of depression and a higher risk of mortality [6].

To overcome the above-mentioned negative consequences of a sedentary lifestyle, participating in physical exercise activities has proven to be an effective non-pharmacological strategy for reducing fat mass accumulation and the associated diseases [7-9]. There are general guidelines that promote the prescription of 30-60-min moderate-intensity exercise 3 times per week [10]. However, these types of recommendations usually fail to produce clear insights regarding the effects that different modes of exercise have on fat mass loss. There is extensive literature recommending aerobic continuous training interventions conducted on treadmills, stationary bikes, and/or rowing ergometers [10]. Although continuous aerobic training presents its potential benefits regarding fat mass loss, it is excessively repetitive and psychologically exhausting when compared with other intermittent training interventions [11, 12]. Also, both continuous and intermittent aerobic exercise modes produce positive effects on human health, with intermittent exercise being the most promising method [13, 14].

Given the highly monotonous aspect of continuous aerobic training, the practice of more enjoyable intermittent aerobic exercise, such as recreational soccer, has been extensively studied regarding its effects on the general health, cardiorespiratory fitness, and motivation of sedentary adults [11, 15-17]. Indeed, a systematic review aiming to analyse the effects of recreational soccer on aerobic fitness and general health in sedentary healthy and unhealthy individuals revealed that this type of physical activity produced significant positive effects on the cardiovascular system and skeletal muscles in both groups [18]. Interestingly, Aslan et al. [19] recently found that young adult sedentary subjects participating in only 1 session per week of recreational soccer exhibited significant decreases in body fat, as well as significant increases in aerobic, power, and strength performance after 8 weeks of the intervention. However, the same study did not determine any adaptation in fat-free mass, heart rate measures, or anaerobic performance, suggesting the need of higher frequency (e.g., 2-3 sessions per week) [19].

Furthermore, as mentioned earlier, recreational soccer has an exponential potential to promote regular exercise in sedentary populations owing to the inherent motivational, social, and competitive aspects of this kind of training intervention $[11,16]$. To date, the overall systematic reviews and meta-analyses have investigated the effects that recreational soccer has on physical fitness and general health parameters $[3,15$, $17,20]$. However, to the best of the authors' knowledge, there is no systematic review with meta-analysis with a focus on the effects of recreational soccer on fat mass changes. For those reasons, the aim of this systematic review with meta-analysis was to assess the impact of recreational soccer on fat mass in untrained sedentary adults.

\section{Material and methods}

The current systematic review and meta-analysis is in line with the recommendations of the Cochrane Collaboration guidelines [21]. The structure of the article follows the PRISMA (Preferred Reporting Items for Systematic Reviews and Meta-Analyses) guidelines [22].

\section{Eligibility criteria}

The eligibility criteria of the systematic review with meta-analysis were based on the PICOS model (Population, Intervention, Comparator, Outcomes, Study design). Table 1 presents the inclusion and exclusion criteria.

\section{Information sources}

Four electronic databases (PubMed, Scopus, SPORTDiscus, and Web of Science) were searched for relevant publications prior to 30 July 2021. After the selection of the articles, a manual search was performed in the reference list of the included articles aiming to retrieve additional studies that might fit the eligibility criteria. The final list of included articles and the eligibility criteria were sent to 2 independent experts in recreational soccer to help identify eventual relevant articles not included in the search. The experts were selected from the list of experts in soccer in the Expertscape website. The search strategy was not provided to the experts in order not to bias them in their searches.

\section{Search strategy}

Keywords and synonyms were entered in various combinations in all fields of the 4 databases: (" "untrained" OR "sedentary”) "adult*”) AND ((“recreational" OR "walk*” OR "medic*") AND ("soccer" OR "football”)) AND ("fat*” OR "body fat” OR "body com- 
Table 1. Inclusion and exclusion criteria for the systematic review

\begin{tabular}{|c|c|c|}
\hline Item & Inclusion criteria & Exclusion criteria \\
\hline Population & $\begin{array}{l}\text { Untrained adults (> } 18 \text { years old), } \\
\text { no restrictions for sex or clinical condition. } \\
\text { Adults were not exposed to specific } \\
\text { pharmacological or diet-oriented plan }\end{array}$ & $\begin{array}{l}\text { Adults exposure to regular exercise. Youth } \\
\text { participants }(<18 \text { years old). Participants } \\
\text { exposed to specific pharmacological or diet- } \\
\text { oriented plan }\end{array}$ \\
\hline Intervention & $\begin{array}{l}\text { Recreational soccer training lasting } \\
\text { for a minimum of } 4 \text { weeks, without restrictions } \\
\text { for weekly frequency }\end{array}$ & $\begin{array}{l}\text { Other team sports (e.g., Australian football, } \\
\text { American football, basketball, volleyball, } \\
\text { handball). Programs with less than } 4 \text { weeks } \\
\text { of training. Combined training (recreational } \\
\text { soccer and other training methods) }\end{array}$ \\
\hline Comparator & Passive or active control groups & $\begin{array}{l}\text { Passive control with evidence of participation } \\
\text { in structured exercise }\end{array}$ \\
\hline Outcomes & $\begin{array}{l}\text { Pre- and post-intervention values } \\
\text { (mean and standard deviation) of fat mass } \\
\text { (direct, indirect, and criterion methods) }\end{array}$ & $\begin{array}{l}\text { Other outcomes not including fat mass } \\
\text { (e.g., skinfold thickness, lean mass, body } \\
\text { mass index); no information on pre- or post- } \\
\text { intervention values (e.g., follow-up excluded) }\end{array}$ \\
\hline Study design & Randomized controlled trials & Non-randomized controlled trials \\
\hline Additional criteria & $\begin{array}{l}\text { Only original and full-text studies written } \\
\text { in English }\end{array}$ & $\begin{array}{l}\text { Written in other language than English. } \\
\text { Article types other than original (e.g., reviews, } \\
\text { letters to editors, trial registrations, proposals } \\
\text { for protocols, editorials, book chapters, } \\
\text { conference abstracts) }\end{array}$ \\
\hline
\end{tabular}

position" OR "health indices") AND ("intervention" OR "RCT" OR "control*” OR "experimental"). The search results were exported to the EndNote software. No filters or limits were used.

\section{Selection process}

FMC and FTGF performed the searches, the removal of duplicates, screening of titles and abstracts, and analysis of the full texts in an independent way. Any disagreements were discussed with a third author (RS).

\section{Data collection process}

The data items were defined before the data extraction, with the aim to avoid bias in the analysis. FMC and FTGF performed the data extraction independently; in cases of discrepancy, a third author (RS) entered the discussion until a consensus was achieved. An Excel datasheet was designed on the basis of the Cochrane Consumers and Communication Review Group's template [23] in order to assess inclusion requirements, and subsequently tested on 10 randomly selected studies (i.e., pilot testing).

\section{Data items}

Only repeated measures (at least pre-post) were considered for data analysing and reporting. Fat mass (\%) was selected as the main outcome. Only fat mass of the total body was considered and extracted. No partial regions were considered. Fat mass was extracted for indirect methods (e.g., anthropometry, bioelectric impedance analysis), direct methods (e.g., total body water, total body counting, neutron activation), and criterion methods (e.g., body density, dual-energy X-ray absorptiometry, computed tomography, magnetic resonance imaging). Additional information about the study was also extracted: (i) participants (e.g., number, age, sex, clinical condition); and (ii) training intervention (duration, weekly frequency, training regimen, intensity).

\section{Assessment of methodological quality}

The Physiotherapy Evidence Database (PEDro) scale was used to assess the methodological quality of the included studies. There are 11 items on the PEDro checklist, but item 1 is not included in the total score. Therefore, the methodological quality of the included 
studies was rated from 0 (lowest quality) to 10 (highest quality). This scale evaluates different aspects of the study design, such as participant eligibility criteria, randomization, blinding, attrition, and reporting of data. The validity and reliability of the PEDro scale has been established previously [24-26]. Additionally, its agreement with other scales (e.g., Cochrane risk of bias tool) has been reported [27]. Moreover, the PEDro scale is probably one of the scales most frequently used in the literature, helping to make comparisons between meta-analyses. In accordance with cut-off scores, the methodological quality was rated as 'poor' $(<4)$, 'fair' (4-5), 'good' (6-8), or 'excellent' (9-10) in some subfields; however, it is not possible to satisfy all scale items in some areas of physiotherapy practice. Moreover, in the context of this study, it is not possible to blind the participants regarding whether they are trained or not, which makes item 5 of the PEDro scale an unfair criterion to assess the methodological quality of studies in our systematic review. Therefore, as outlined in previous systematic reviews in some sub-fields of physiotherapy [28, 29], the methodological quality of studies was interpreted by using the following convention, based on the summary score: studies that scored $\leq 3$ points were considered as being of "poor quality,' studies scoring 4 or 5 points were considered as being of 'moderate quality,' and studies that scored 6-10 points were considered as being of 'high quality'. Two authors performed the methodological quality assessment independently. Disagreements in the evaluations between the reviewers were resolved through discussion and consensus.

Summary measures, synthesis of results, and publication bias

A meta-analysis was carried out only in the case of having 3 or more studies providing baseline and follow-up data for the same measure. Pre-training and post-training mean and standard deviation $(S D)$ for dependent variables were used to calculate effect sizes (ES; Hedges' $g$ ) for each outcome measure in the recreation soccer training and control groups. Data were standardized with post-intervention $S D$ values. The random-effects model was used to account for differences between studies that might impact on the small-sided game (SSG)-based effect [36, 37]. The $E S$ values are presented with $95 \%$ confidence intervals (CI). The calculated $E S$ values were interpreted by using the following scale: < 0.2, trivial; 0.2-0.6, small; > 0.6-1.2, moderate; > 1.2-2.0, large; > 2.0-4.0, very large; $>4.0$, extremely large [38]. Heterogeneity was assessed with the $I^{2}$ statistics, with values of $<25 \%$, $25-75 \%$, and $>75 \%$ considered to represent low, moderate, and high levels of heterogeneity, respectively [39]. The risk of bias was explored by using the extended Egger's test [40]. When bias was present, the trim and fill method was applied [41], with L0 assumed as the default estimator for missing studies [42]. All analyses were carried out with the Comprehensive MetaAnalysis software (version 2; Biostat, Englewood, NJ, USA). Statistical significance was set at $p \leqslant 0.05$.

\section{Ethical approval}

The conducted research is not related to either human or animal use.

\section{Results}

\section{Study selection}

The initial search made in the databases retrieved 4565 titles (SportDISCUS: 411; PubMed: 21; Web of Science: 11; Scopus: 4122). Those titles were exported to the EndNote ${ }^{\mathrm{TM}}$ reference manager (X9; Clarivate Analytics, Philadelphia, PA, USA). The duplicates ( $n=$ 2362) were removed automatically and manually. In addition, there were records marked as ineligible by automation tools $(n=7)$. The remaining 2196 articles were screened for their relevance on the basis of the abstract and title, which led to the removal of another 2158 studies. The full texts of the remaining 38 articles were examined diligently; after reading them, further 16 studies were excluded for the following reasons (R): R1, no fat mass: 5; R2, youth participants: 4; R3, no recreational soccer: 2; R4, footballers: 1 ; R5, acute responses: 1; R6, cross-sectional study: 1; R7, cohort study: $1 ; \mathrm{R} 8$, calorie restricted diet: 1 (more information: Figure 1). The remaining 22 articles were included in the systematic review, providing mean and $S D$ post-training data for the main outcome.

\section{Study characteristics}

The characteristics of the 22 included studies can be found in Table 2 . The included randomized controlled studies involve 22 experimental (soccer) groups with 777 participants, 9 active control groups with 158 participants, and 21 passive control groups with a total of 728 participants.

The characteristics of the recreational soccer interventions and control groups are presented in Tables 3 and 4, respectively. 


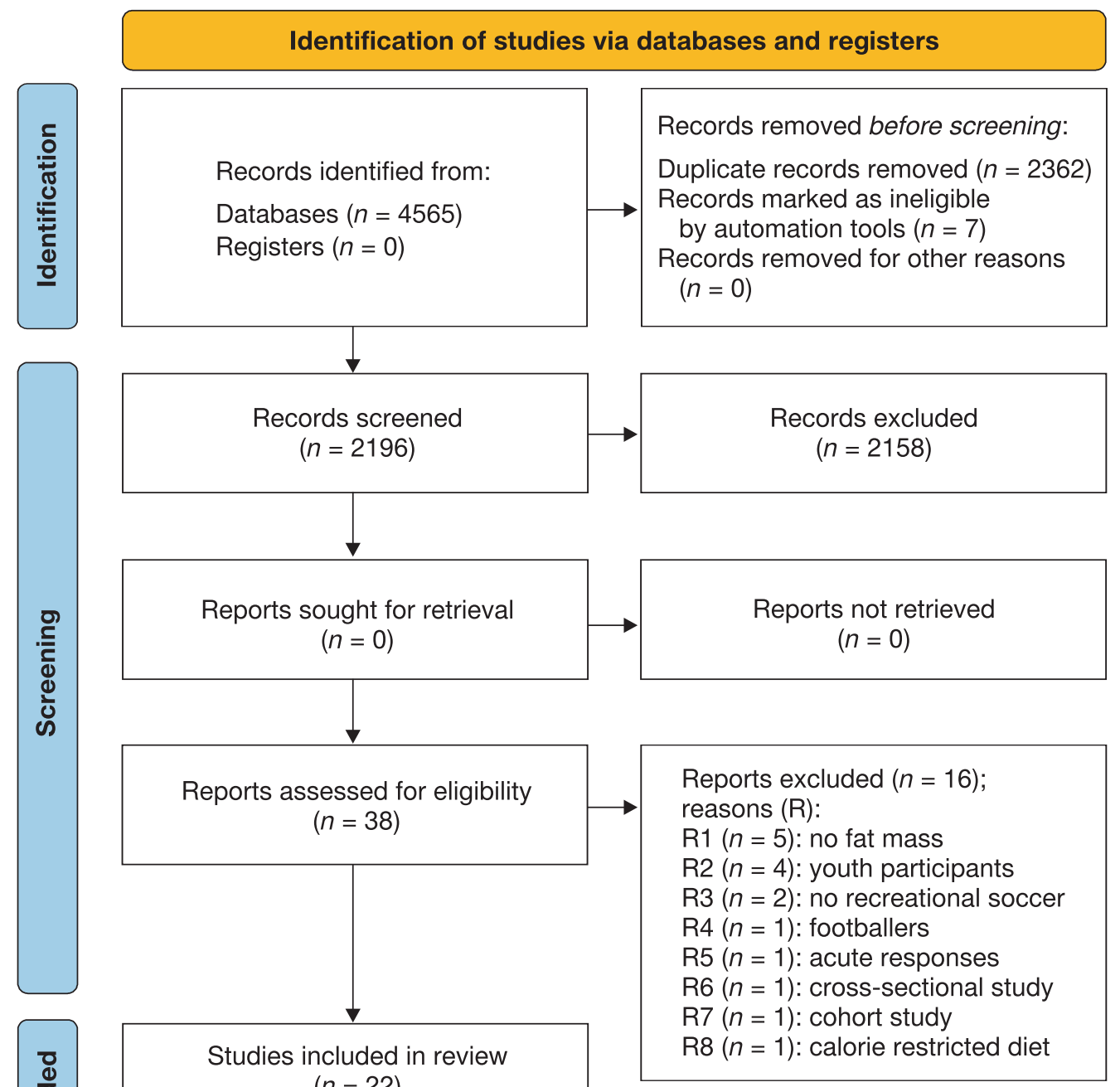

Methodological quality of the studies

The included studies were scored on the basis of the PEDro checklist (Table 5). The mean score for the 22 included studies was $6.81 \pm 0.58$ (minimum: 6 ; maximum: 8). It was observed that the studies had 'good' methodological quality.

\section{Results of syntheses}

Table 6 presents the summary of results of total fat mass ( $\mathrm{kg}$ ) or relative fat mass (\%) reported before and after the recreational soccer intervention, as well as in the active control and passive control groups in the included articles.

Overall, 9 studies provided fat mass data involving 9 soccer training groups and 9 active control groups compared (pooled $n=350$ ). The results (Fig- ure 2) showed similar changes in fat mass after soccer training and among active controls $(E S=-0.070$, trivial; $95 \%$ CI: -0.28 to $0.14 ; p=0.505 ; I^{2}=0.0 \%$; Egger's test $p=0.986$ ).

Regarding interventions with males (3 study groups; $E S=-0.24 ; 95 \%$ CI: -0.68 to $0.21 ; p=0.301$; withingroup $\left.I^{2}=0.0 \%\right)$ and those with females (6 study groups; $E S=-0.03$; $95 \%$ CI: -0.26 to $0.21 ; p=0.834$; within-group $I^{2}=0.0 \%$ ), both sex groups achieved a non-significant (and similar, i.e., $p=0.412$ between sexes) reduction of fat mass after soccer training compared with active controls.

A total of 21 studies provided fat mass data involving 21 soccer training groups and 21 passive control groups compared (pooled $n=1000$ ). The results (Figure 3) showed a greater change in fat mass after soccer training than among passive controls $(E S=-0.43$, small; $95 \%$ CI: -0.59 to $-0.27 ; p<0.001$; $I^{2}=35.0 \%$; Egger's test $p=0.652$ ). 
Table 2. Characteristics of the included studies

\begin{tabular}{|c|c|c|c|c|c|c|c|}
\hline Study & $n$ & Mean age (years) & Sex & Population & $\begin{array}{l}\text { Type of control } \\
\text { group }\end{array}$ & $\begin{array}{l}\text { Instrument } \\
\text { used for } \\
\text { measuring fat } \\
\text { mass }\end{array}$ & $\begin{array}{l}\text { Outcome } \\
\text { extracted }\end{array}$ \\
\hline
\end{tabular}

\begin{tabular}{|c|c|c|c|c|c|c|c|}
\hline $\begin{array}{l}\text { Krustrup } \\
\text { et al. [30] }\end{array}$ & $\begin{array}{l}\text { Soccer group: } 13 \\
\text { Running group: } 12 \\
\text { Passive control: } 11\end{array}$ & $\begin{array}{l}\text { Soccer group: } 30 \pm 2 \\
\text { Running group: } 31 \pm 2 \\
\text { Passive control: } 30 \pm 2\end{array}$ & Men & Healthy & $\begin{array}{c}1 \text { active (running) } \\
1 \text { passive }\end{array}$ & DXA & $\begin{array}{l}\text { Fat mass } \\
\text { total }(\mathrm{kg})\end{array}$ \\
\hline
\end{tabular}

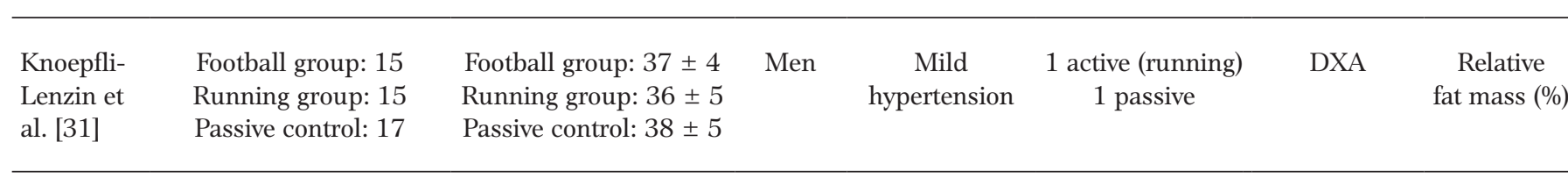

\begin{tabular}{|c|c|c|c|c|c|c|c|}
\hline $\begin{array}{l}\text { Krustrup } \\
\text { et al. [32] }\end{array}$ & $\begin{array}{l}\text { Football group: } 25 \\
\text { Running group: } 25 \\
\text { Passive control: } 15\end{array}$ & $\begin{array}{l}\text { Football group: } 37 \pm 2 \\
\text { Running group: } 37 \pm 1 \\
\text { Passive control: } 33 \pm 2\end{array}$ & Women & Healthy & $\begin{array}{c}1 \text { active (running) } \\
1 \text { passive }\end{array}$ & DXA & $\begin{array}{c}\text { Relative } \\
\text { fat mass (\%) }\end{array}$ \\
\hline
\end{tabular}

\begin{tabular}{lclccc}
\hline & & & & & \\
Krustrup & Football group: 9 & Football group: $40 \pm 3$ & Women & Healthy & 1 active (running) \\
et al. [33] & Running group: 10 & Running group: $40 \pm 2$ & & 1 passive \\
& Passive control: 9 & Passive control: $38 \pm 4$ & & fat mass (\%)
\end{tabular}

\begin{tabular}{lccccc}
\hline $\begin{array}{l}\text { Randers } \\
\text { et al. [45] }\end{array}$ & $\begin{array}{c}\text { Football group: } 10 \\
\text { Passive control: } 7\end{array}$ & All: 20-43 & Men & Healthy & $\begin{array}{c}\text { Relative } \\
\text { fat mass (\%) }\end{array}$ \\
\hline $\begin{array}{l}\text { Randers } \\
\text { et al. [46] }\end{array}$ & $\begin{array}{c}\text { Soccer group: } 22 \\
\text { Passive control: } 10\end{array}$ & $\begin{array}{c}\text { Soccer group: } 37 \pm 10 \\
\text { Passive control: } 43 \pm 9\end{array}$ & Men & Homeless & $\begin{array}{c}\text { DX } \\
\text { passive }\end{array}$ \\
\hline
\end{tabular}

\begin{tabular}{|c|c|c|c|c|c|c|c|}
\hline $\begin{array}{l}\text { Gray et al. } \\
{[47]}\end{array}$ & $\begin{array}{l}\text { Intervention group: } 51 \\
\text { Comparison group: } 52\end{array}$ & $\begin{array}{c}\text { Intervention group: } \\
48.2 \pm 8.4 \\
\text { Comparison group: } \\
45.9 \pm 8.4\end{array}$ & Men & $\begin{array}{l}\text { Overweight } \\
\text { and obese }\end{array}$ & 1 passive & $\begin{array}{c}\text { Electronic } \\
\text { bioimpedance }\end{array}$ & $\begin{array}{c}\text { Relative } \\
\text { fat mass (\%) }\end{array}$ \\
\hline $\begin{array}{l}\text { Krustrup } \\
\text { et al. [48] }\end{array}$ & $\begin{array}{c}\text { Soccer group: } 22 \\
\text { Doctor's advice group: } 11\end{array}$ & All: $31-54$ & Men & $\begin{array}{c}\text { Mild-to- } \\
\text { moderate } \\
\text { hypertension }\end{array}$ & 1 passive & DXA & $\begin{array}{c}\text { Relative } \\
\text { fat mass (\%) }\end{array}$ \\
\hline
\end{tabular}

\begin{tabular}{|c|c|c|c|c|c|c|c|}
\hline $\begin{array}{l}\text { Andersen } \\
\text { et al. [49] }\end{array}$ & $\begin{array}{l}\text { Football group: } 12 \\
\text { Passive control: } 9\end{array}$ & $\begin{array}{l}\text { Football group: } \\
50.6 \pm 7.1 \\
\text { Passive control: } \\
48.7 \pm 9.2\end{array}$ & Men & $\begin{array}{c}\text { Type } 2 \text { diabetes } \\
\text { mellitus }\end{array}$ & 1 passive & DXA & $\begin{array}{c}\text { Fat mass - } \\
\text { total }(\mathrm{kg})\end{array}$ \\
\hline $\begin{array}{l}\text { Barene } \\
\text { et al. [34] }\end{array}$ & $\begin{array}{c}\text { Soccer group: } 37 \\
\text { Zumba group: } 35 \\
\text { Passive control: } 35\end{array}$ & $\begin{array}{c}\text { Soccer group: } 44.1 \pm 8.7 \\
\text { Zumba group: } \\
45.9 \pm 9.6 \\
\text { Passive control: } \\
47.4 \pm 9.5\end{array}$ & Women & ND & $\begin{array}{c}1 \text { active (Zumba) } \\
1 \text { passive }\end{array}$ & DXA & $\begin{array}{c}\text { Relative } \\
\text { fat mass (\%) }\end{array}$ \\
\hline $\begin{array}{l}\text { Barene } \\
\text { et al. [35] }\end{array}$ & $\begin{array}{c}\text { Soccer group: } 24 \\
\text { Zumba group: } 25 \\
\text { Passive control: } 25\end{array}$ & $\begin{array}{c}\text { Soccer group: } 44.1 \pm 8.7 \\
\text { Zumba group: } \\
45.9 \pm 9.6 \\
\text { Passive control: } \\
47.4 \pm 9.5\end{array}$ & Women & ND & $\begin{array}{c}1 \text { active (Zumba) } \\
1 \text { passive }\end{array}$ & DXA & $\begin{array}{c}\text { Relative } \\
\text { fat mass }(\%)\end{array}$ \\
\hline
\end{tabular}


F.M. Clemente et al., Effects of recreational soccer on fat mass

\begin{tabular}{|c|c|c|c|c|c|c|c|}
\hline $\begin{array}{l}\text { Connolly } \\
\text { et al. [36] }\end{array}$ & $\begin{array}{l}\text { Soccer group: } 13 \\
\text { Vibration group: } 17 \\
\text { Passive control: } 14\end{array}$ & $\begin{array}{c}\text { Soccer group: } 39 \pm 6 \\
\text { Vibration group: } 40 \pm 4 \\
\text { Passive control: } 40 \pm 5\end{array}$ & Women & Healthy & $\begin{array}{c}1 \text { active } \\
\text { (vibration) } \\
1 \text { passive }\end{array}$ & DXA & $\begin{array}{c}\text { Relative } \\
\text { fat mass }(\%\end{array}$ \\
\hline
\end{tabular}

\begin{tabular}{|c|c|c|c|c|c|c|c|}
\hline $\begin{array}{l}\text { De Sousa } \\
\text { et al. [50] }\end{array}$ & $\begin{array}{c}\text { Football + diet: } 19 \\
\text { Diet: } 15\end{array}$ & All: 48-68 & $\begin{array}{c}\text { Men } \\
\text { and } \\
\text { women }\end{array}$ & $\begin{array}{l}\text { Type } 2 \text { diabetes } \\
\text { mellitus }\end{array}$ & 1 passive & DXA & $\begin{array}{c}\text { Relative } \\
\text { fat mass (\%) }\end{array}$ \\
\hline
\end{tabular}

\begin{tabular}{|c|c|c|c|c|c|c|c|}
\hline $\begin{array}{l}\text { Mohr et al. } \\
\text { [51] }\end{array}$ & $\begin{array}{l}\text { Football group: } 21 \\
\text { Control group: } 20\end{array}$ & $\begin{array}{l}\text { Football group: } 45 \pm 3 \\
\text { Control group: } 43 \pm 3\end{array}$ & Women & $\begin{array}{c}\text { Mild } \\
\text { hypertension }\end{array}$ & 1 passive & DXA & $\begin{array}{c}\text { Fat mass - } \\
\text { total }(\mathrm{kg})\end{array}$ \\
\hline $\begin{array}{l}\text { Uth et al. } \\
\text { [52] }\end{array}$ & $\begin{array}{l}\text { Football group: } 23 \\
\text { Control group: } 26\end{array}$ & $\begin{array}{c}\text { Football group: } \\
67.1 \pm 7.1 \\
\text { Control group: } \\
66.5 \pm 4.9\end{array}$ & Men & Prostate cancer & 1 passive & DXA & $\begin{array}{c}\text { Relative } \\
\text { fat mass (\%) }\end{array}$ \\
\hline $\begin{array}{l}\text { Andersen } \\
\text { et al. [38] }\end{array}$ & $\begin{array}{l}\text { Football group: } 9 \\
\text { Resistance group: } 9 \\
\text { Control group: } 8\end{array}$ & $\begin{array}{c}\text { Football group: } \\
68.0 \pm 4.0 \\
\text { Resistance group: } \\
69.1 \pm 3.1 \\
\text { Control group: } \\
67.4 \pm 2.7\end{array}$ & Men & Healthy & $\begin{array}{c}1 \text { active } \\
\text { (resistance) } \\
1 \text { passive }\end{array}$ & DXA & $\begin{array}{c}\text { Relative fat } \\
\text { mass (\%) }\end{array}$ \\
\hline
\end{tabular}

\begin{tabular}{lcccccc}
\hline $\begin{array}{l}\text { Krustrup } \\
\text { et al. [53] }\end{array}$ & $\begin{array}{c}\text { Soccer group: } 19 \\
\text { Control group: } 12\end{array}$ & $\begin{array}{c}\text { Soccer group: } 45 \pm 6 \\
\text { Control group: } 45 \pm 4\end{array}$ & Women & $\begin{array}{c}\text { Mild } \\
\text { hypertension }\end{array}$ & 1 passive & $\begin{array}{c}\text { DXA } \\
\text { Relative } \\
\text { fat mass (\%) }\end{array}$ \\
\hline $\begin{array}{l}\text { Uth et al. } \\
{[54]}\end{array}$ & $\begin{array}{l}\text { Football group: } 21 \\
\text { Control group: } 20\end{array}$ & $\begin{array}{c}\text { Football group: } \\
67.1 \pm 7.1 \\
\text { Control group: } \\
66.5 \pm 4.9\end{array}$ & Men & Prostate cancer & 1 passive & $\begin{array}{c}\text { DXA } \\
\text { Fat mass }- \\
\text { total (kg) }\end{array}$ \\
\hline
\end{tabular}

\begin{tabular}{|c|c|c|c|c|c|c|c|}
\hline $\begin{array}{l}\text { Reddy et } \\
\text { al. [43] }\end{array}$ & $\begin{array}{l}\text { Football group: } 11 \\
\text { Control group: } 9\end{array}$ & All: 50-65 & $\begin{array}{l}\text { Men and } \\
\text { women }\end{array}$ & ND & 1 passive & $\begin{array}{c}\text { Electronic } \\
\text { bioimpedance }\end{array}$ & $\begin{array}{c}\text { Relative } \\
\text { fat mass (\%) }\end{array}$ \\
\hline $\begin{array}{l}\text { Hunt et al. } \\
{[55]}\end{array}$ & $\begin{array}{c}\text { Football Fans } \\
\text { in Training: } 374 \\
\text { Comparison group: } 373\end{array}$ & $\begin{array}{c}\text { Football Fans } \\
\text { in Training: } 47.0 \pm 8.07 \\
\text { Comparison group: } \\
47.2 \pm 7.89\end{array}$ & Men & $\begin{array}{l}\text { Overweight } \\
\text { and obese }\end{array}$ & 1 passive & $\begin{array}{c}\text { Electronic } \\
\text { bioimpedance }\end{array}$ & $\begin{array}{c}\text { Relative } \\
\text { fat mass (\%) }\end{array}$ \\
\hline $\begin{array}{l}\text { Aslan et al. } \\
\text { [19] }\end{array}$ & $\begin{array}{l}\text { Soccer group: } 10 \\
\text { Control group: } 10\end{array}$ & $\begin{array}{l}\text { Soccer group: } \\
22.1 \pm 1.8 \\
\text { Control group: } \\
23.4 \pm 1.6\end{array}$ & Men & Healthy & 1 passive & $\begin{array}{l}\text { Skinfold } \\
\text { thickness }\end{array}$ & $\begin{array}{c}\text { Relative } \\
\text { fat mass (\%) }\end{array}$ \\
\hline $\begin{array}{l}\text { Ortiz et al. } \\
{[37]}\end{array}$ & $\begin{array}{l}\text { Soccer group: } 17 \\
\text { Running group: } 10\end{array}$ & $\begin{array}{c}\text { Soccer group: } \\
28.8 \pm 3.6 \\
\text { Running group: } \\
27.1 \pm 4.7\end{array}$ & Women & Healthy & 1 active (running) & $\begin{array}{l}\text { Skinfold } \\
\text { thickness }\end{array}$ & $\begin{array}{c}\text { Relative } \\
\text { fat mass (\%) }\end{array}$ \\
\hline
\end{tabular}

ND - not described, DXA - dual-energy X-ray absorptiometry 


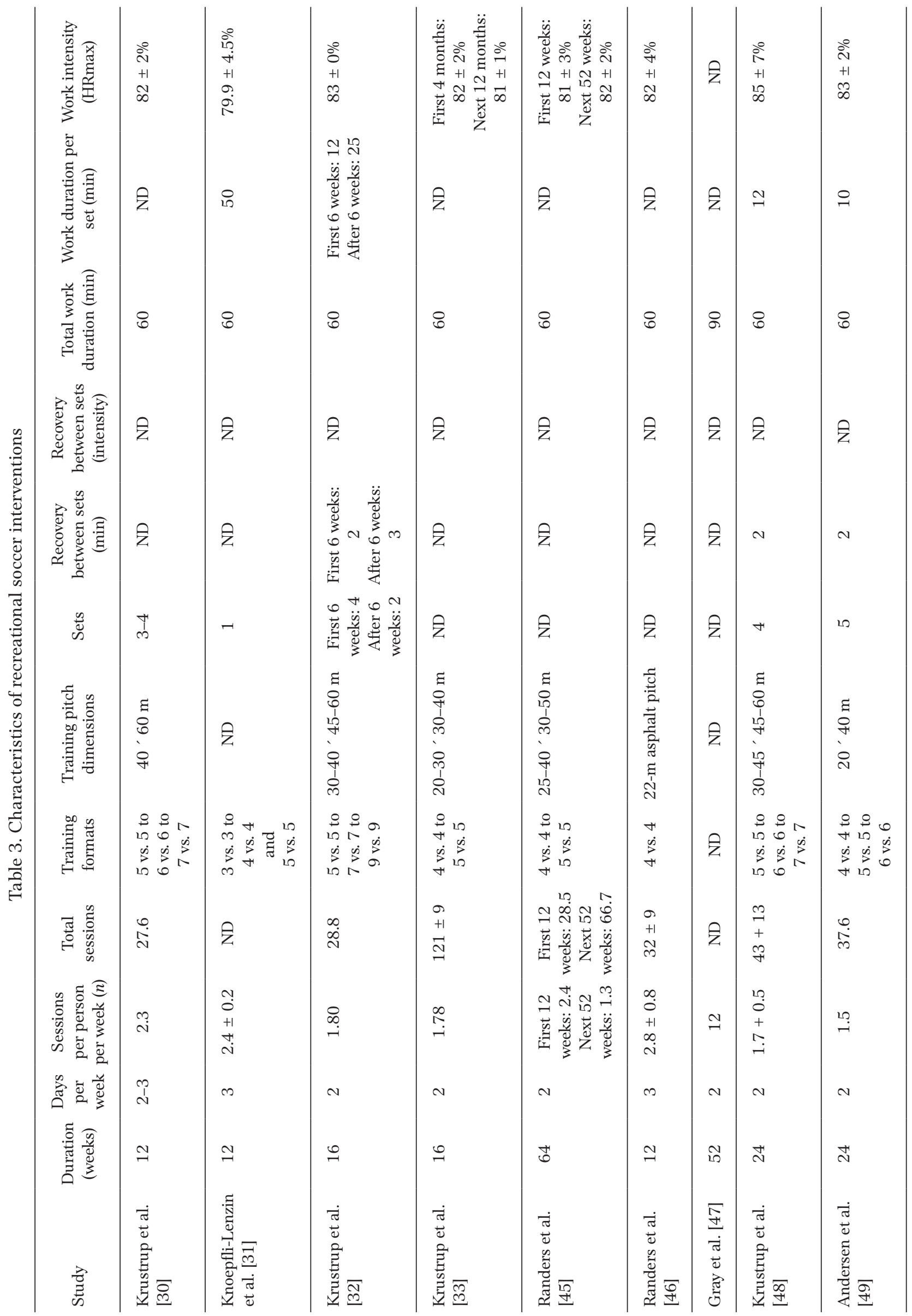




\begin{tabular}{|c|c|c|c|c|c|c|c|c|c|c|c|c|}
\hline $\begin{array}{l}\stackrel{\circ}{\circ} \\
+ \\
+ \\
+1 \\
0 \\
0 \\
0 \\
0\end{array}$ & $\begin{array}{l}\text { iे } \\
i \\
+1 \\
+0 \\
0 \\
\infty \\
i\end{array}$ & $\begin{array}{l}\stackrel{0}{10} \\
+1 \\
10 \\
\infty\end{array}$ & $\begin{array}{l}80 \\
0 \\
0 \\
0 \\
0 \\
8\end{array}$ & 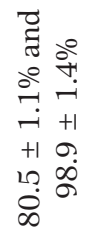 & $\begin{array}{l}\text { Oें } \\
\dot{0} \\
\dot{+}\end{array}$ & 穵 & $\begin{array}{l}\sigma^{0} \\
10 \\
+1 \\
+1 \\
\infty\end{array}$ & 合 & 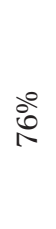 & 合 & $\begin{array}{l}\stackrel{0}{9} \\
+ \\
+1 \\
0 \\
\dot{\infty}\end{array}$ & $\begin{array}{l}\text { oे } \\
0 \\
\infty\end{array}$ \\
\hline 至 & $\stackrel{\stackrel{2}{N}}{ }$ & $\stackrel{20}{\sim}$ & $\approx$ & 会 & $\stackrel{10}{\longrightarrow}$ & $\stackrel{2}{\sim}$ & $\approx$ & $\stackrel{20}{\longrightarrow}$ & $\begin{array}{l}0 \\
10 \\
10 \\
1\end{array}$ & 令 & 令 & 合 \\
\hline 8 & 80 & $\stackrel{10}{\longrightarrow}$ & 아 & 8 & 8 & 8 & 8 & $\begin{array}{l}8 \\
1 \\
1\end{array}$ & 8 & \& & 8 & 10 \\
\hline 用 & 1 & 立 & 立 & 立 & 学 & 立 & 立 & 字 & 合 & 羊 & 学 & 令 \\
\hline 合 & ما & 立 & $\infty$ & 合 & 立 & $N$ & 合 & 学 & 令 & 立 & 学 & 令 \\
\hline 合 & $\sim$ & - & $N$ & 令 & 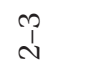 & $\underbrace{+1}_{d}$ & $H$ & 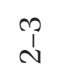 & - & 空 & 要 & 会 \\
\hline 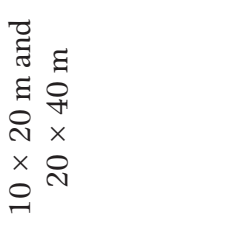 & 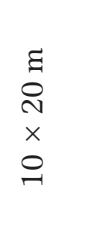 & 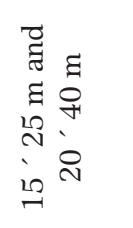 & 令 & 穵 & 令 & $\begin{array}{l}\Xi \\
0 \\
0 \\
1 \\
1 \\
1 \\
\text { in } \\
7 \\
0 \\
0\end{array}$ & 令 & 安 & 穴 & 合 & 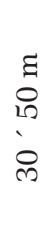 & 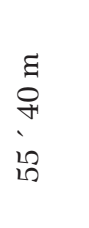 \\
\hline 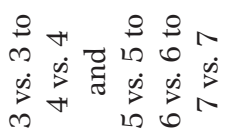 & 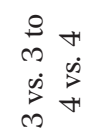 & 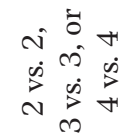 & 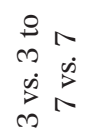 & 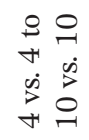 & 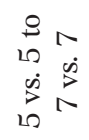 & 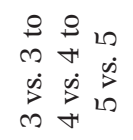 & 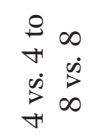 & 合 & $\begin{array}{l}10 \\
\dot{0} \\
\dot{2} \\
\dot{2}\end{array}$ & 文 & 叜 & 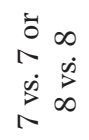 \\
\hline $\begin{array}{l}3 \\
\dot{0} \\
+1 \\
0 \\
\dot{D} \\
\stackrel{\infty}{0}\end{array}$ & 1 & 穵 & s & $\begin{array}{l}-7 \\
+1 \\
\stackrel{+}{+}\end{array}$ & $\stackrel{\infty}{\sim}$ & 至 & $\begin{array}{l}\stackrel{N}{ } \\
+1 \\
\infty \\
\stackrel{N}{N}\end{array}$ & 至 & 空 & 至 & 至 & 立 \\
\hline 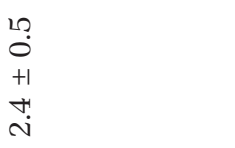 & $\begin{array}{l}\infty \\
\infty \\
\infty \\
+1 \\
\forall \\
10 \\
10\end{array}$ & 立 & 立 & $\begin{array}{l}\overrightarrow{0} \\
+1 \\
+1 \\
\dot{\infty}\end{array}$ & 空 & $\begin{array}{l}\infty \\
0 \\
+1 \\
\stackrel{+}{-}\end{array}$ & $\begin{array}{l}J \\
0 \\
+1 \\
b \\
i \\
N\end{array}$ & $\begin{array}{l}0 \\
\stackrel{1}{1} \\
o \\
0 \\
0\end{array}$ & 穴 & $\stackrel{\sim}{\sim}$ & 文 & $\infty$ \\
\hline$\stackrel{q}{d}$ & $\stackrel{p}{\sim}$ & $\sim$ & $\infty$ & $\infty$ & 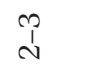 & 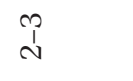 & 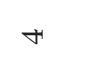 & 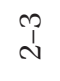 & - & $N$ & - & $\infty$ \\
\hline$\stackrel{\sim}{\sim}$ & 우 & $\stackrel{\bullet}{\sim}$ & $\cong$ & $\stackrel{10}{\sim}$ & $\cong$ & N & กิ & $\widetilde{\infty}$ & $\stackrel{\sim}{\sim}$ & กิ & $\infty$ & $\infty$ \\
\hline 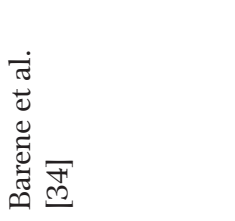 & 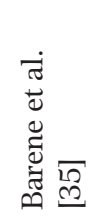 & 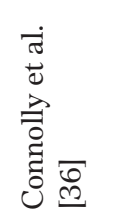 & 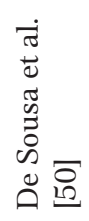 & 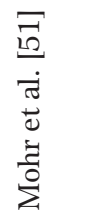 & 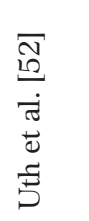 & 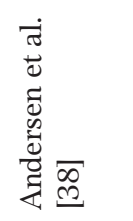 & 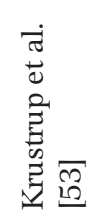 & 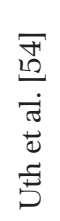 & 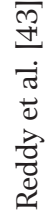 & 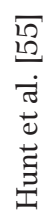 & 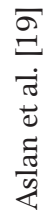 & 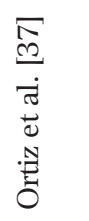 \\
\hline
\end{tabular}




\section{HUMAN MOVEMENT}

F.M. Clemente et al., Effects of recreational soccer on fat mass

Table 4. Characteristics of control groups

\begin{tabular}{ll}
\hline Study & Active control Passive control
\end{tabular}

\section{Krustrup et al. [30] Participants completed 3-4 sets within 1 hour of running, 2 or 3 times per week for 12 weeks, with an average intensity of $82 \% \mathrm{HRmax}$ \\ Continued daily life activities}

Knoepfli-Lenzin et al. [31]

Running group completed a 1-hour training session 2.5 times per week for 12 weeks. The mean training intensity was $79.4 \pm 1.3 \%$

Krustrup et al. [32] Running group performed 5 min of low-intensity warm-up (walking and jogging), followed by four 12-min sessions consisting of moderate-intensity continuous running (80-84\% of their HRmax), twice a week for 16 weeks. After the 6 weeks, all runners were able to run for 55 min continuously

Krustrup et al. [33] One hour of running 2 times a week for 16 months. The running speed was individually adjusted to elicit $81 \%$ HRmax during the first 4 weeks, and $82 \%$ in the last 12 months
Maintained their sedentary lifestyle

Performed no physical training, only measurements were made (before and after 16 weeks)

Performed no physical training, continued their usual daily life activities

\begin{tabular}{|c|c|c|}
\hline Randers et al. [45] & - & $\begin{array}{l}\text { Instructed to remain physically inactive over } \\
\text { the first } 12 \text { weeks. No restrictions were applied } \\
\text { during the last } 52 \text {-week period }\end{array}$ \\
\hline
\end{tabular}

Randers et al. [46]

Gray et al. [47]

Krustrup et al. [48]
$-$

$-$

Continued their normal routines

No details were given. Only, the comparison group received the Football Fans in Training program 4 months after the intervention group

Doctor's advice group received traditional physician-guided recommendations (favourable effects of healthy lifestyle, recommended physical activity, nutrition) on cardiovascular risk factor modification

Andersen et al. [49]

$-$

Inactive; no details

Barene et al. [34] Zumba group: continuous dance movements to

No details; only measurements were made Latin music with varying intensity, $2-3$ sessions (1 hour) per week for 12 weeks. Average heart rate during training was $136 \pm 17 \mathrm{bpm}$ or $75.3 \pm 7.1 \%$ HRmax

Barene et al. [35]

Zumba group: continuous dance movement (1 hour) using Latin music with varying intensity, twice a week throughout the 40 weeks. The average heart rate during the 1-hour training sessions was $136 \pm 17$, which corresponds to $74.9 \pm 7.2 \%$ HRmax
Only measurements were made; no intervention was applied 
Vibration group performed 15-min training sessions (7 exercises) with average heart rates of $90 \mathrm{bpm}$, twice per a week for 16 weeks. The protocol consisted of a 3-min warm-up at a frequency of $6 \mathrm{~Hz}$ with an amplitude of $2 \mathrm{~mm}$. Each 1 -min bout was followed by $1 \mathrm{~min}$ of recovery. The frequency and amplitude were increased in certain weeks
De Sousa et al. [50]
Continued their normal daily lives
Diet group: received dietary counselling for weight reduction, 500-1000 kcal of energy intake per day. The prescribed diet was balanced and rich in fibre (minimum of $20 \mathrm{~g} /$ day). The diet consisted of $45-60 \%$ carbohydrate, $15-20 \%$ protein, and 20-30\% fat
Mohr et al. [51]

th et al. [52]
Inactive: had no training or lifestyle changes during the 15-week period

Encouraged to maintain their baseline physical activity level

Andersen et al. [38] Resistance training: 5-min low-intensity warm-

Inactive; no details

Inactive; no details
up, followed by leg press, seated leg extension, hamstring curl, pull-down, and lateral dumbbell raises. Sets were interspaced by 1.5 -min rest, and at the end of the session, 5-min core training was made. Exercise progressed: $3 \times 16-20 \mathrm{RM}$ (weeks 0-4), $3 \times 12 \mathrm{RM}$ (weeks 5-8), $3 \times 10 \mathrm{RM}$ (weeks 9-12), and $4 \times 8 \mathrm{RM}$ (weeks 13-52)

\begin{tabular}{|c|c|c|}
\hline Krustrup et al. [53] & - & $\begin{array}{l}\text { Inactive: did not take part in physical training } \\
\text { and were encouraged not to implement lifestyle } \\
\text { changes during the } 1 \text {-year period }\end{array}$ \\
\hline Uth et al. [54] & - & $\begin{array}{l}\text { Encouraged to maintain their habitual level } \\
\text { of physical activity }\end{array}$ \\
\hline Reddy et al. [43] & - & No details \\
\hline Hunt et al. [55] & - & $\begin{array}{l}\text { No details were given. Only, comparison group } \\
\text { put on a 12-month waiting list, the Football Fans } \\
\text { in Training program applied after the } 12 \text { months }\end{array}$ \\
\hline Aslan et al. [19] & - & Continued their routine daily lives \\
\hline Ortiz et al. [37] & $\begin{array}{l}\text { Running group: } 50 \text { min } 3 \text { times a week for } \\
8 \text { weeks ( } 5 \text { min at an intensity corresponding } \\
\text { to onset of blood lactate accumulation) ( } 85.7 \% \text { ), } \\
\text { with passive recovery periods of } 1 \text { min for } 2 \text { days. } \\
\text { For one day, they practised continuously for } \\
50 \text { min at the intensity corresponding to lactate } \\
\text { threshold ( } 67.3-70.5 \%) \text { The average heart rate } \\
\text { during the training period: } 82.2 \% \text { HRmax }\end{array}$ & - \\
\hline
\end{tabular}

HRmax - maximal heart rate, RM - repetition maximum 
F.M. Clemente et al., Effects of recreational soccer on fat mass

Study name

Andersen et al. 「491

Barene et al. $\lceil 341$

Barene et al. 「351

Connollv et al. $\lceil 361$

Knoepfli-Lenzin et al. ¡311

Krustrup et al. ¡30]

Krustrup et al. $\lceil 32\rceil$

Krustrun et al. $\lceil 331$

Ortiz et al. [37]

$\begin{array}{cc}\begin{array}{c}\text { Hedges's } \\ \text { g }\end{array} & \begin{array}{c}\text { Standard } \\ \text { error }\end{array} \\ -0.162 & 0.450 \\ -0.067 & 0.233 \\ 0.017 & 0.223 \\ -0.327 & 0.361 \\ -0.136 & 0.356 \\ -0.411 & 0.391 \\ -0.203 & 0.279 \\ 0.081 & 0.439 \\ 0.594 & 0.395 \\ -0.070 & 0.105\end{array}$

$\underline{\text { Statistics for each study }}$

Variance $\begin{gathered}\text { Lower } \\ \text { limit }\end{gathered} \begin{gathered}\text { Upper } \\ \text { limit }\end{gathered}$

$\begin{array}{lll}0.202 & -1.044 & 0.719\end{array}$

$\begin{array}{lll}0.054 & -0.524 & 0.390 \\ 0.050 & -0.420 & 0.454\end{array}$

$\begin{array}{lll}0.050 & -0.420 & 0.454\end{array}$

$\begin{array}{lll}0.130 & -1.034 & 0.381\end{array}$

$\begin{array}{lll}0.127 & -0.833 & 0.561\end{array}$

$\begin{array}{lll}0.153 & -1.178 & 0.356\end{array}$

$\begin{array}{lll}0.078 & -0.750 & 0.345\end{array}$

$\begin{array}{lll}0.193 & -0.780 & 0.942\end{array}$

$\begin{array}{lll}0.156 & -0.180 & 1.368\end{array}$

$\begin{array}{lll}0.011 & -0.277 & 0.136\end{array}$
Hedges's g and 95\% CI

Z-Value p-Value

$\begin{array}{ll}-0.360 & 0.719\end{array}$

$-0.287 \quad 0.774$

$0.077 \quad 0.939$

$\begin{array}{ll}-0.905 & 0.366\end{array}$

$-0.382 \quad 0.703$

$\begin{array}{ll}-1.050 & 0.294\end{array}$

$-0.726 \quad 0.468$

$0.185 \quad 0.854$

$1.504 \quad 0.133$

$-0.666 \quad 0.505$

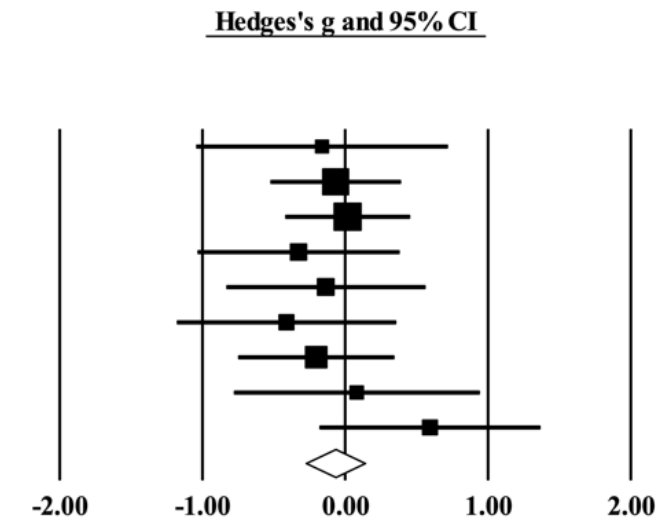

Favours soccer Favours active control

Figure 2. Changes (reduction) in fat mass after soccer training compared with active controls.

Values shown are effect sizes (Hedges' g) with 95\% confidence intervals (CI). The size of the plotted squares reflects the statistical relative weight of the study. The white diamond reflects the overall result

\section{Study name \\ Study name}

Andersen et al. [49]

Andersen et al. 「381

Aslan et al. [19]

Barene et al. 「341

Barene et al. 1351

Connollv et al. [361

Gray et al. [47]

Hunt et al. [55]

Knoepfli-Lenzin et al. [31]

Krustrup et al. $\lceil 301$

Krustrup et al. 「32]

Krustrup et al. $\lceil 331$

Krustrup et al. $\lceil 481$

Krustrun et al. 「531

Mohr et al. $\lceil 511$

Randers et al. [45]

Randers et al. 「461

Reddy et al. [43]

Sousa et al. $\lceil 501$

Uth et al. [52]

Uth et al. [54]

\section{Statistics for each study}

\section{Hedges's Standard Lower Upper}

g error Variance limit limit $Z$-Value p-Value

$\begin{array}{rrrrrrr}-0.415 & 0.428 & 0.183 & -1.254 & 0.424 & -0.969 & 0.332 \\ -0.919 & 0.487 & 0.238 & -1.874 & 0.036 & -1.886 & 0.059 \\ -0.122 & 0.429 & 0.184 & -0.963 & 0.718 & -0.285 & 0.776 \\ -0.173 & 0.234 & 0.055 & -0.631 & 0.285 & -0.739 & 0.460 \\ -0.192 & 0.222 & 0.049 & -0.627 & 0.243 & -0.864 & 0.388 \\ -0.199 & 0.374 & 0.140 & -0.933 & 0.535 & -0.531 & 0.595 \\ -0.713 & 0.202 & 0.041 & -1.108 & -0.317 & -3.532 & 0.000 \\ -0.407 & 0.074 & 0.005 & -0.551 & -0.262 & -5.507 & 0.000 \\ -0.336 & 0.348 & 0.121 & -1.018 & 0.345 & -0.967 & 0.334 \\ -0.854 & 0.414 & 0.172 & -1.666 & -0.042 & -2.062 & 0.039 \\ -0.805 & 0.333 & 0.111 & -1.457 & -0.154 & -2.422 & 0.015 \\ -0.386 & 0.454 & 0.206 & -1.275 & 0.503 & -0.851 & 0.395 \\ -0.253 & 0.362 & 0.131 & -0.962 & 0.455 & -0.701 & 0.484 \\ -1.870 & 0.431 & 0.185 & -2.714 & -1.026 & -4.344 & 0.000 \\ -1.050 & 0.328 & 0.107 & -1.692 & -0.408 & -3.206 & 0.001 \\ -0.796 & 0.487 & 0.237 & -1.751 & 0.159 & -1.633 & 0.102 \\ -0.290 & 0.374 & 0.140 & -1.023 & 0.442 & -0.778 & 0.437 \\ 0.223 & 0.432 & 0.187 & -0.623 & 1.070 & 0.517 & 0.605 \\ 0.000 & 0.337 & 0.114 & -0.661 & 0.661 & 0.000 & 1.000 \\ -0.184 & 0.282 & 0.080 & -0.737 & 0.369 & -0.651 & 0.515 \\ -0.027 & 0.306 & 0.094 & -0.627 & 0.574 & -0.087 & 0.931 \\ -0.428 & 0.082 & 0.007 & -0.589 & -0.268 & -5.227 & 0.000\end{array}$

Hedges's g and $95 \%$ CI

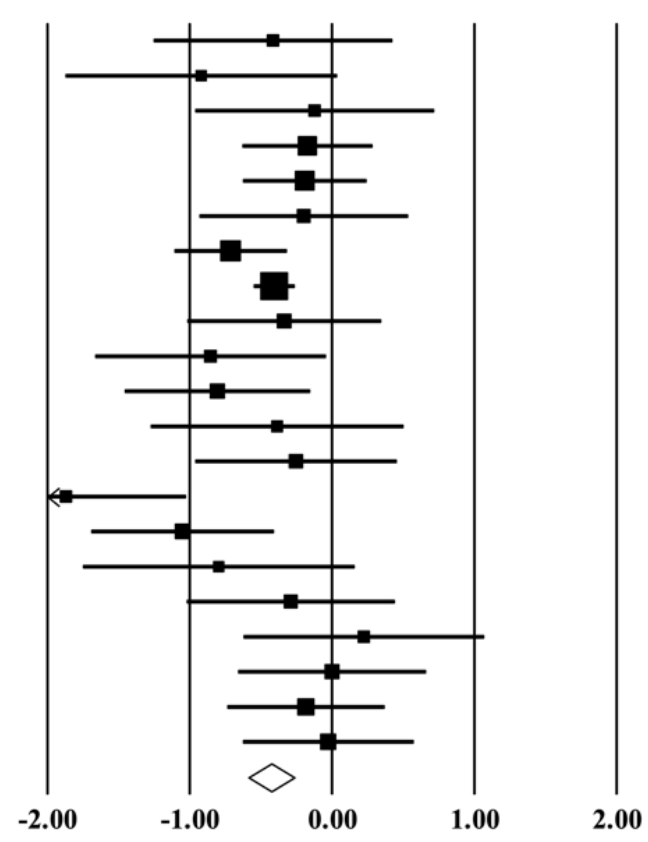

Favours soccer Favours passive control

Figure 3. Changes (reduction) in fat mass after soccer training compared with passive controls.

Values shown are effect sizes (Hedges' g) with 95\% confidence intervals (CI). The size of the plotted squares reflects the statistical relative weight of the study. The white diamond reflects the overall result 
Table 6. Summary of the included studies and results of fat mass before and after intervention

\begin{tabular}{|c|c|c|c|c|c|c|c|}
\hline Study & Group & Population & Sex & $n$ & $\begin{array}{c}\text { Before } \\
(\text { mean } \pm S D)\end{array}$ & $\begin{array}{c}\text { After } \\
(\text { mean } \pm S D)\end{array}$ & $\begin{array}{c}\text { After - before } \\
(\Delta \%)\end{array}$ \\
\hline Krustrup et al. [30] & SG & $\mathrm{H}$ & M & 13 & $19.9 \pm 2.4$ & $17.2 \pm 2.1$ & -13.5 \\
\hline Knoepfli-Lenzin et al. [31] & SG & $\mathrm{C}$ & M & 15 & $29.2 \pm 4.3$ & $27.2 \pm 4.3$ & -6.8 \\
\hline Krustrup et al. [32] & SG & $\mathrm{H}$ & $\mathrm{W}$ & 25 & $35.8 \pm 1.2$ & $33.8 \pm 1.3$ & -5.5 \\
\hline Krustrup et al. [33] & SG & $\mathrm{H}$ & $\mathrm{W}$ & 9 & $34.4 \pm 1.8$ & $32.7 \pm 1.9$ & -4.9 \\
\hline Randers et al. [45] & SG & $\mathrm{H}$ & $\mathrm{M}$ & 10 & $23.6 \pm 2.6$ & $19.8 \pm 2.5$ & -16.1 \\
\hline Randers et al. [46] & SG & Homeless & M & 22 & $14.1 \pm 7.1$ & $12.4 \pm 6.5$ & -12.0 \\
\hline Gray et al. [47] & SG & $\mathrm{C}$ & $\mathrm{M}$ & 51 & $30.8 \pm 3.8$ & $29.4 \pm 4.0$ & -4.5 \\
\hline Krustrup et al. [48] & SG & $\mathrm{C}$ & M & 22 & $30.7 \pm 4.9$ & $28.5 \pm 4.2$ & -7.1 \\
\hline Andersen et al. [49] & SG & $\mathrm{C}$ & M & 12 & $29.5 \pm 2.0$ & $27.8 \pm 2.0$ & -5.7 \\
\hline Barene et al. [34] & SG & ND & $\mathrm{W}$ & 37 & $34.7 \pm 6.1$ & $33.6 \pm 6.2$ & -3.1 \\
\hline Barene et al. [35] & SG & ND & $\mathrm{W}$ & 41 & $34.7 \pm 6.1$ & $33.5 \pm 6.0$ & -3.4 \\
\hline Connolly et al. [36] & SG & $\mathrm{H}$ & $\mathrm{W}$ & 13 & $37.4 \pm 6.9$ & $35.7 \pm 6.2$ & -4.5 \\
\hline De Sousa et al. [50] & SG & $\mathrm{C}$ & $\mathrm{M} / \mathrm{W}$ & 19 & $34.6 \pm 1.6$ & $32.2 \pm 1.5$ & -6.9 \\
\hline Mohr et al. [51] & SG & $\mathrm{C}$ & $\mathrm{W}$ & 21 & $36.6 \pm 2.0$ & $34.3 \pm 2.0$ & -6.2 \\
\hline Uth et al. [52] & SG & $\mathrm{C}$ & $\mathrm{M}$ & 23 & $32.6 \pm 5.8$ & $31.7 \pm 5.6$ & -2.7 \\
\hline Andersen et al. [38] & SG & $\mathrm{H}$ & M & 9 & $27.2 \pm 2.3$ & $25.6 \pm 2.4$ & -5.8 \\
\hline Krustrup et al. [53] & SG & $\mathrm{C}$ & $\mathrm{W}$ & 19 & $35.8 \pm 2.2$ & $32.2 \pm 2.0$ & -10.5 \\
\hline Uth et al. [54] & SG & $\mathrm{C}$ & $\mathrm{M}$ & 21 & $26.8 \pm 7.8$ & $26.5 \pm 8.4$ & -1.1 \\
\hline Reddy et al. [43] & SG & ND & $\mathrm{M} / \mathrm{W}$ & 11 & $27.1 \pm 7.3$ & $30.9 \pm 19.6$ & 14.0 \\
\hline Hunt et al. [55] & SG & $\mathrm{C}$ & $\mathrm{M}$ & 374 & $31.8 \pm 5.7$ & $29.6 \pm 5.6$ & -6.9 \\
\hline Aslan et al. [19] & SG & $\mathrm{H}$ & $\mathrm{M}$ & 10 & $13.2 \pm 2.1$ & $12.7 \pm 2.3$ & -3.7 \\
\hline Ortiz et al. [37] & SG & $\mathrm{H}$ & $\mathrm{W}$ & 17 & $27.2 \pm 4.4$ & $27.4 \pm 4.5$ & 0.7 \\
\hline Krustrup et al. [30] & $\mathrm{AC}$ & $\mathrm{H}$ & M & 12 & $20.7 \pm 2.7$ & $19.0 \pm 2.6$ & -8.2 \\
\hline Knoepfli-Lenzin et al. [31] & $\mathrm{AC}$ & $\mathrm{C}$ & $\mathrm{M}$ & 15 & $29.7 \pm 4.1$ & $28.3 \pm 4.3$ & -4.7 \\
\hline Krustrup et al. [32] & $\mathrm{AC}$ & $\mathrm{H}$ & $\mathrm{W}$ & 25 & $32.6 \pm 1.7$ & $30.9 \pm 1.6$ & -5.2 \\
\hline Krustrup et al. [33] & $\mathrm{AC}$ & $\mathrm{H}$ & $\mathrm{W}$ & 10 & $34.9 \pm 2.4$ & $33.0 \pm 2.7$ & -5.4 \\
\hline Barene et al. [34] & $\mathrm{AC}$ & ND & $\mathrm{W}$ & 35 & $36.0 \pm 5.8$ & $35.3 \pm 5.6$ & -1.9 \\
\hline Barene et al. [35] & $\mathrm{AC}$ & ND & $\mathrm{W}$ & 38 & $36.0 \pm 5.8$ & $34.7 \pm 5.5$ & -3.6 \\
\hline Connolly et al. [36] & $\mathrm{AC}$ & $\mathrm{H}$ & $\mathrm{W}$ & 17 & $38.9 \pm 6.9$ & $39.3 \pm 6.3$ & 1.0 \\
\hline Ortiz et al. [37] & $\mathrm{AC}$ & $\mathrm{H}$ & $\mathrm{W}$ & 10 & $27.1 \pm 4.3$ & $24.5 \pm 4.7$ & -9.5 \\
\hline Andersen et al. [38] & $\mathrm{AC}$ & $\mathrm{H}$ & $\mathrm{M}$ & 9 & $29.4 \pm 2.3$ & $28.2 \pm 2.3$ & -4.0 \\
\hline Krustrup et al. [30] & PC & $\mathrm{H}$ & M & 11 & $19.6 \pm 3.3$ & $19.3 \pm 3.3$ & -1.5 \\
\hline Knoepfli-Lenzin et al. [31] & $\mathrm{PC}$ & $\mathrm{C}$ & M & 17 & $29.7 \pm 7.1$ & $29.8 \pm 7.3$ & 0.3 \\
\hline Krustrup et al. [32] & PC & $\mathrm{H}$ & $\mathrm{W}$ & 15 & $28.8 \pm 1.6$ & $28.0 \pm 1.7$ & -2.7 \\
\hline Krustrup et al. [33] & $\mathrm{PC}$ & $\mathrm{H}$ & $\mathrm{W}$ & 9 & $29.4 \pm 2.7$ & $28.6 \pm 2.5$ & -2.7 \\
\hline Randers et al. [45] & $\mathrm{PC}$ & $\mathrm{H}$ & $\mathrm{M}$ & 7 & $24.4 \pm 4.3$ & $23.8 \pm 5.2$ & -2.4 \\
\hline Randers et al. [46] & $\mathrm{PC}$ & Homeless & M & 10 & $17.4 \pm 8.8$ & $18.0 \pm 10.0$ & 3.4 \\
\hline Gray et al. [47] & PC & $\mathrm{C}$ & $\mathrm{M}$ & 52 & $30.7 \pm 5.4$ & $32.8 \pm 5.6$ & 6.8 \\
\hline Krustrup et al. [48] & $\mathrm{PC}$ & $\mathrm{C}$ & M & 11 & $30.9 \pm 7.0$ & $29.9 \pm 5.4$ & -3.2 \\
\hline Andersen et al. [49] & PC & $\mathrm{C}$ & M & 9 & $31.0 \pm 5.3$ & $30.9 \pm 5.2$ & -0.3 \\
\hline Barene et al. [34] & $\mathrm{PC}$ & ND & $\mathrm{W}$ & 35 & $36.3 \pm 6.4$ & $36.3 \pm 6.4$ & 0.0 \\
\hline Barene et al. [35] & $\mathrm{PC}$ & ND & $\mathrm{W}$ & 39 & $36.3 \pm 6.4$ & $36.3 \pm 6.4$ & 0.0 \\
\hline Connolly et al. [36] & $\mathrm{PC}$ & $\mathrm{H}$ & $\mathrm{W}$ & 14 & $35.4 \pm 8.2$ & $35.2 \pm 8.2$ & -0.5 \\
\hline De Sousa et al. [50] & $\mathrm{PC}$ & $\mathrm{C}$ & $\mathrm{M} / \mathrm{W}$ & 15 & $37.5 \pm 1.8$ & $35.1 \pm 1.8$ & -6.4 \\
\hline Mohr et al. [51] & $\mathrm{PC}$ & $\mathrm{C}$ & W & 20 & $33.0 \pm 1.6$ & $32.6 \pm 1.5$ & -1.2 \\
\hline Uth et al. [52] & PC & $\mathrm{C}$ & $\mathrm{M}$ & 26 & $32.9 \pm 5.2$ & $32.9 \pm 4.0$ & 0.0 \\
\hline Andersen et al. [38] & $\mathrm{PC}$ & $\mathrm{H}$ & $\mathrm{M}$ & 8 & $29.4 \pm 1.8$ & $29.8 \pm 1.6$ & 1.3 \\
\hline Krustrup et al. [53] & $\mathrm{PC}$ & $\mathrm{C}$ & $\mathrm{W}$ & 12 & $30.4 \pm 2.0$ & $30.5 \pm 1.8$ & 0.1 \\
\hline Uth et al. [54] & $\mathrm{PC}$ & $\mathrm{C}$ & M & 20 & $30.2 \pm 6.0$ & $30.1 \pm 6.1$ & -0.3 \\
\hline Reddy et al. [43] & $\mathrm{PC}$ & ND & $\mathrm{M} / \mathrm{W}$ & 9 & $31.1 \pm 5.7$ & $31.3 \pm 7.5$ & 0.6 \\
\hline Hunt et al. [55] & $\mathrm{PC}$ & $\mathrm{C}$ & $\mathrm{M}$ & 373 & $31.5 \pm 5.2$ & $31.5 \pm 5.2$ & 0.0 \\
\hline Aslan et al. [19] & $\mathrm{PC}$ & $\mathrm{H}$ & $\mathrm{M}$ & 10 & $12.7 \pm 2.3$ & $12.5 \pm 2.4$ & -1.57 \\
\hline
\end{tabular}

$\Delta \%$ - percentage changes representing the mean differences (after - before), SG - soccer group, AC - active control, PC - passive control, $\mathrm{H}$ - healthy, C - clinical, ND - not described, $\mathrm{W}$ - women, $\mathrm{M}$ - men 
Regarding interventions with males (12 study groups; $E S=-0.42$; $95 \%$ CI: -0.54 to $-0.30 ; p<0.001$; within-group $\left.I^{2}=0.0 \%\right)$ and those with females (7 study groups; $E S=-0.62 ; 95 \%$ CI: -1.04 to $-0.20 ; p=0.004$; within-group $I^{2}=67.4 \%$ ), both sex groups achieved a significant (and similar, i.e., $p=0.362$ between sexes) reduction of fat mass after soccer training compared with passive controls.

Regarding interventions with healthy participants (7 study groups; $E S=-0.58$; $95 \%$ CI: -0.88 to -0.27 ; $p<0.001$; within-group $\left.I^{2}=0.0 \%\right)$ and those with a clinical condition (10 study groups; $E S=-0.49 ; 95 \%$ CI: -0.74 to $-0.22 ; p<0.001$; within-group $I^{2}=57.6 \%$ ), both groups achieved a significant (and similar, i.e., $p=0.662$ between groups) reduction of fat mass after soccer training compared with passive controls.

\section{Discussion}

This systematic review with meta-analysis aimed to analyse the effects of recreational soccer on fat mass in untrained sedentary adults. A meta-analysis for control groups was conducted. The results showed that fat mass was significantly reduced after recreational soccer interventions compared with passive control groups. However, there were no significant differences in fat mass reduction between recreational soccer and active control groups.

\section{Recreational soccer vs. active control groups}

Regarding the included recreational soccer and other exercise activities (active controls) interventions, both groups demonstrated similar effects in fat mass reduction, without significant differences between them. The lack of differences between both groups in fat mass changes suggests that participation in different physical exercise interventions produces similar body fat mass changes. From the 9 included studies that used active control groups, 8 [30-37] involved continuous aerobic activities, such as continuous running or Zumba. Only 1 study [38] implemented resistance training, which has a more intermittent nature. Given that, these findings corroborate the potential of continuous and intermittent exercise interventions to induce significant improvements in general health [8, 13]. Although the overall result, shown in Figure 2, reveals a lack of differences between the groups, the studies together tend to favour the recreational soccer interventions. Indeed, this tendency was previously reported for intermittent and continuous physical activities [13].
Furthermore, both males and females exhibited similar, not significantly different reductions in fat mass after recreational soccer interventions compared with the active groups. The lack of significant differences between recreational soccer and active groups in fat mass changes after the training interventions may be due to the more controlled exercise intensity in the active groups, as the studies involved continuous running and individualized the running intensity. In fact, Krustrup et al. [33] adjusted all training sessions intensity to $81 \%$ and $82 \%$ of maximal heart rate measure to maintain a consistent intensity during 16 months. Also, given the high intensity of soccer displacements, it may be possible that participating in only 2-3 sessions per week produces physiological adaptations similar to those with aerobic continuous stimulus in just 8 weeks of practice [37].

However, the recreational soccer groups are expected to present more intra- and inter-subject variations in exercise intensity during the interventions [39]. Given that, it seems that a more longitudinal recreational soccer approach (i.e., more than 12 weeks of practice) is required to produce greater changes (reduction) in fat mass [32]. Despite the similarities of fat mass changes between the groups, recreational soccer interventions have the advantage of being more enjoyable and less monotonous [17, 40]. Indeed, untrained populations would benefit from the regular practice of a recreational team sport as their maintenance in such interventions is more effective than in endurance interventions. This maintenance is usually related to the psychosocial development and community integration [41].

\section{Recreational soccer vs. passive control groups}

There were significant differences in fat mass changes between recreational soccer and passive control groups, independently of sex and population (healthy or with clinical conditions). Indeed, extensive literature exists regarding positive effects that participation in regular physical exercise has on sedentary population's general health [42]. However, to the best of our knowledge, there is no systematic review with meta-analysis focused on the influence of recreational soccer on fat mass changes over time. The findings of the present study suggest the effectiveness of regular participation in recreational soccer to significantly reduce the fat mass of untrained sedentary populations.

In fact, the included studies generally showed significant reductions in fat mass after a period of the recreational soccer intervention. In contrast, one study 
reported increases in fat mass after 12 weeks of recreational soccer intervention [43]. However, in that study, the authors used a 'walking football' 5 vs. 5 SSG only once a week, and they affirmed that their study revealed a lack of positive impact on fitness and body composition. This corroborates the need to implement more regular sessions per week, with activities of a higher intensity than walking [20]. Interestingly, a relatively recent systematic review of systematic reviews that analysed the relationships between physical activity and health status demonstrated that it was not "evidence-based' to recommend a given threshold of weekly physical activity volume [42]. The authors of the abovementioned systematic review of systematic reviews based that statement on the fact that minimal physical activity (very low weekly volume) produced relative risk reductions and, consequently, positive health adaptations in untrained sedentary individuals [42].

Despite that, sedentary populations seem to benefit from more regular participation in weekly physical activities, aiming to produce more than minimal positive general health and fitness adaptations over time [15]. In fact, a study conducted among 114 healthy individuals revealed that although weekly volumes of physical exercise below the international recommendations produced minimal health benefits, larger volumes presented greater improvements in body composition, aerobic capacity, and musculoskeletal system [44].

\section{Limitations, future research, and practical implications}

The studies included in the present systematic review with meta-analysis involved some limitations. Primarily, the limitations were as follows: (i) limited sample sizes of the overall studies; (ii) lower number of studies with active control groups; (iii) the use of different SSG protocols (different pitch dimensions and numbers of players), with no distinction between them regarding the effects of fat mass changes; (iv) not focusing on fat mass changes; and (v) the use of different activities in active control groups. Future studies should focus on the effects of longitudinal and more consistent recreational soccer interventions on body composition parameters. Also, analysing the differences between SSG protocols (e.g., 3v3, 5v5, or 7v7) in terms of fat mass changes would be interesting.

Despite the limitations of the included studies, the present systematic review with meta-analysis revealed some practical implications. A main one refers to the implementation of recreational soccer as an effective way to reduce fat mass in untrained sedentary indi- viduals. The lack of differences between recreational soccer and other traditional/analytical aerobic continuous interventions is of great importance in clinical practice as individuals who have a preference for practising team sports can expect similar effective reductions of fat mass. Also, these reductions occur independently of sex and clinical condition, which reinforces the benefits of regular participation in recreational soccer in cases of individuals with mild-tomoderate hypertension, type 2 diabetes mellitus, prostate cancer, and overweight or obesity. Furthermore, the practice of recreational soccer presents the advantage of being a more enjoyable, fun, and motivational intervention than traditional aerobic continuous training.

\section{Conclusions}

This systematic review and meta-analysis investigated the effects of recreational soccer on fat mass changes in untrained sedentary adults compared with active and passive controls. There were no significant differences between recreational soccer and active control groups in fat mass changes after the training interventions. However, fat mass was significantly reduced after recreational soccer interventions compared with passive control groups. This systematic review with meta-analysis highlights the benefits and greater pertinence of regular participation in recreational soccer to reduce fat mass and increase the motivation to be active when compared with other traditional training interventions, such as continuous running.

\section{Funding}

The study received no external funding.

\section{Acknowledgements}

This work was supported by Fundação para a Ciência e Tecnologia/Ministério da Ciência, Tecnologia e Ensino Superior through national funds and, when applicable, co-funded by European Union funds under the project UIDB/EEA/50008/2020.

\section{Disclosure statement}

No author has any financial interest or received any financial benefit from this research.

\section{Conflict of interest}

The authors state no conflict of interest.

\section{References}

1. Kyle UG, Morabia A, Schutz Y, Pichard C. Sedentarism affects body fat mass index and fat-free mass index in 
adults aged 18 to 98 years. Nutrition. 2004;20(3):255260; doi: 10.1016/j.nut.2003.11.019.

2. Bowden Davies KA, Pickles S, Sprung VS, Kemp GJ, Alam U, Moore DR, et al. Reduced physical activity in young and older adults: metabolic and musculoskeletal implications. Ther Adv Endocrinol Metab. 2019;10: 2042018819888824; doi:10.1177/2042018819888824.

3. Sarmento H, Clemente FM, Marques A, Milanovic Z, Harper LD, Figueiredo A. Recreational football is medicine against non-communicable diseases: a systematic review. Scand J Med Sci Sports. 2020;30(4):618-637; doi: 10.1111/sms.13611.

4. Goossens GH. The metabolic phenotype in obesity: fat mass, body fat distribution, and adipose tissue function. Obes Facts. 2017;10(3):207-215; doi: 10.1159/00047 1488.

5. Gamboa-Gómez CI, Simental-Mendía LE, RodríguezMorán M, Guerrero-Romero F. The fat-to-lean mass ratio, a novel anthropometric index, is associated to glucose metabolic disorders. Eur J Intern Med. 2019; 63:74-78; doi: 10.1016/j.ejim.2019.03.017.

6. Blasco BV, García-Jiménez J, Bodoano I, GutiérrezRojas L. Obesity and depression: its prevalence and influence as a prognostic factor: a systematic review. Psychiatry Investig. 2020;17(8):715-724; doi: 10.30773/ pi.2020.0099.

7. Yin Y-H, Liu JYW, Välimäki M. Effectiveness of nonpharmacological interventions on the management of sarcopenic obesity: a systematic review and meta-analysis. Exp Gerontol. 2020;135:110937; doi: 10.1016/j. exger.2020.110937.

8. Willis LH, Slentz CA, Bateman LA, Shields AT, Piner LW, Bales CW, et al. Effects of aerobic and/or resistance training on body mass and fat mass in overweight or obese adults. J Appl Physiol. 2012;113(12):1831-1837; doi: 10.1152/japplphysiol.01370.2011.

9. Can S, Arslan E, Biernat E, Piątkowska M. Different physical activity guidelines and its association with socio-demographic and anthropometric characteristics among working women. Balt J Health Phys Act. 2017;9(3):115-123; doi: 10.29359/BJHPA.09.3.11.

10. Barrow DR, Abbate LM, Paquette MR, Driban JB, Vincent HK, Newman C, et al. Exercise prescription for weight management in obese adults at risk for osteoarthritis: synthesis from a systematic review. BMC Musculoskelet Disord. 2019;20(1):610; doi: 10.1186/ s12891-019-3004-3.

11. Castagna C, de Sousa M, Krustrup P, Kirkendall DT. Recreational team sports: the motivational medicine. J Sport Health Sci. 2018;7(2):129-131; doi: 10.1016/j. jshs.2017.12.001.

12. Soylu Y, Arslan E, Sogut M, Kilit B, Clemente FM. Effects of self-paced high-intensity interval training and moderate-intensity continuous training on the physical performance and psychophysiological responses in recreationally active young adults. Biol Sport. 2021;38(4): 555-562; doi: 10.5114/biolsport.2021.100359.
13. Leal JM, Galliano LM, Del Vecchio FB. Effectiveness of high-intensity interval training versus moderate-intensity continuous training in hypertensive patients: a systematic review and meta-analysis. Curr Hypertens Rep. 2020;22(3):26; doi: 10.1007/s11906-020-1030-z.

14. Arslan E, Can S, Demirkan E. Effect of short-term aerobic and combined training program on body composition, lipids profile and psychological health in premenopausal women. Sci Sports. 2017;32(2):106-113; doi: 10.1016/j.scispo.2016.11.004.

15. Luo H, Newton RU, Ma'ayah F, Galvão DA, Taaffe DR. Recreational soccer as sport medicine for middle-aged and older adults: a systematic review. BMJ Open Sport Exerc Med. 2018;4(1):e000336; doi: 10.1136/bmjsem2017-000336.

16. Milanović Z, Pantelić S, Čović N, Sporiš G, Mohr M, Krustrup P. Broad-spectrum physical fitness benefits of recreational football: a systematic review and metaanalysis. Br J Sports Med. 2019;53(15):926-939; doi: 10.1136/bjsports-2017-097885.

17. Milanović Z, Pantelić S, Čović N, Sporiš G, Krustrup P. Is recreational soccer effective for improving $\mathrm{VO}_{2}$ max? A systematic review and meta-analysis. Sports Med. 2015;45(9):1339-1353; doi: 10.1007/s40279-015-0361-4.

18. Hammami A, Chamari K, Slimani M, Shephard RJ, Yousfi N, Tabka Z, et al. Effects of recreational soccer on physical fitness and health indices in sedentary healthy and unhealthy subjects. Biol Sport. 2016;33(2):127137; doi: 10.5604/20831862.1198209.

19. Aslan A, Salci Y, Guvenc A. The effects of weekly recreational soccer intervention on the physical fitness level of sedentary young men. Monten J Sports Sci Med. 2019;8(1):51-59; doi: 10.26773/mjssm.190308.

20. Krustrup P, Aagaard P, Nybo L, Petersen J, Mohr M, Bangsbo J. Recreational football as a health promoting activity: a topical review. Scand J Med Sci Sports. 2010;20(Suppl.1):1-13; doi:10.1111/j.1600-0838.2010. 01108.x.

21. Green S, Higgins J (eds.). Cochrane handbook for systematic reviews of interventions. Hoboken: Wiley; 2005.

22. Moher D, Liberati A, Tetzlaff J, Altman DG, PRISMA Group. Preferred reporting items for systematic reviews and meta-analyses: the PRISMA statement. PLoS Med. 2009;6(7):e1000097; doi: 10.1371/journal.pmed.100 0097.

23. Cochrane Consumers and Communication. Data extraction template for included studies. 2016.

24. De Morton NA. The PEDro scale is a valid measure of the methodological quality of clinical trials: a demographic study. Aust J Physiother. 2009;55(2):129-133; doi: 10.1016/S0004-9514(09)70043-1.

25. Maher CG, Sherrington C, Herbert RD, Moseley AM, Elkins M. Reliability of the PEDro scale for rating quality of randomized controlled trials. Phys Ther. 2003;83(8): 713-721; doi: 10.1093/PTJ/83.8.713.

26. Yamato TP, Maher C, Koes B, Moseley A. The PEDro scale had acceptably high convergent validity, construct 
validity, and interrater reliability in evaluating methodological quality of pharmaceutical trials. J Clin Epidemiol. 2017;86:176-181; doi: 10.1016/j.jclinepi.2017. 03.002 .

27. Moseley AM, Rahman P, Wells GA, Zadro JR, Sherrington C, Toupin-April K, et al. Agreement between the Cochrane risk of bias tool and Physiotherapy Evidence Database (PEDro) scale: a meta-epidemiological study of randomized controlled trials of physical therapy interventions. PLoS One. 2019;14(9):e0222770; doi: 10. 1371/journal.pone.0222770.

28. Stojanović E, Ristić V, McMaster DT, Milanović Z. Effect of plyometric training on vertical jump performance in female athletes: a systematic review and meta-analysis. Sports Med. 2017;47(5):975-986; doi: 10.1007/ s40279-016-0634-6.

29. Ramirez-Campillo R, Gentil P, Negra Y, Grgic J, Girard O. Effects of plyometric jump training on repeated sprint ability in athletes: a systematic review and meta-analysis. Sports Med. 2021;51(10):2165-2179; doi: 10.1007/s40279-021-01479-w.

30. Krustrup P, Nielsen JJ, Krustrup BR, Christensen JF, Pedersen H, Randers MB, et al. Recreational soccer is an effective health-promoting activity for untrained men. Br J Sports Med. 2009;43(11):825-831; doi: 10.1136/ bjsm.2008.053124.

31. Knoepfli-Lenzin C, Sennhauser C, Toigo M, Boutellier U, Bangsbo J, Krustrup P, et al. Effects of a 12-week intervention period with football and running for habitually active men with mild hypertension. Scand J Med Sci Sports. 2010;20(Suppl. 1):72-79; doi: 10.1111/ j.1600-0838.2009.01089.x.

32. Krustrup P, Hansen PR, Randers MB, Nybo L, Martone D, Andersen LJ, et al. Beneficial effects of recreational football on the cardiovascular risk profile in untrained premenopausal women. Scand J Med Sci Sports. 2010;20(Suppl. 1):40-49; doi: 10.1111/j.16000838.2010.01110.x.

33. Krustrup P, Hansen PR, Andersen LJ, Jakobsen MD, Sundstrup E, Randers MB, et al. Long-term musculoskeletal and cardiac health effects of recreational football and running for premenopausal women. Scand J Med Sci Sports. 2010;20(Suppl. 1):58-71; doi: 10.1111/ j.1600-0838.2010.01111.x.

34. Barene S, Krustrup P, Jackman SR, Brekke OL, Holtermann A. Do soccer and Zumba exercise improve fitness and indicators of health among female hospital employees? A 12-week RCT. Scand J Med Sci Sports. 2014;24(6):990-999; doi: 10.1111/sms.12138.

35. Barene S, Krustrup P, Brekke OL, Holtermann A. Soccer and Zumba as health-promoting activities among female hospital employees: a 40-weeks cluster randomised intervention study. J Sports Sci. 2014;32(16): 1539-1549; doi: 10.1080/02640414.2014.906043.

36. Connolly LJ, Scott S, Mohr M, Ermidis G, Julian R, Bangsbo J, et al. Effects of small-volume soccer and vibration training on body composition, aerobic fitness, and muscular $\mathrm{PCr}$ kinetics for inactive women aged 20-45. J Sport Health Sci. 2014;3(4):284-292; doi: 10.1016/j.jshs.2014.07.003.

37. Ortiz JG, da Silva JF, Carminatti LJ, Guglielmo LGA, Diefenthaeler F. Effect of 8 weeks soccer training on health and physical performance in untrained women. J Sports Sci Med. 2018;17(1):17-23.

38. Andersen TR, Schmidt JF, Pedersen MT, Krustrup P, Bangsbo J. The effects of 52 weeks of soccer or resistance training on body composition and muscle function in +65-year-old healthy males - a randomized controlled trial. PLoS One. 2016;11(2):e0148236; doi: 10.1371/journal.pone.0148236.

39. Younesi S, Rabbani A, Clemente FM, Silva R, Sarmento H, Figueiredo AJ. Relationships between aerobic performance, hemoglobin levels, and training load during small-sided games: a study in professional soccer players. Front Physiol. 2021;12:649870; doi: 10.3389/ fphys.2021.649870.

40. Jung ME, Bourne JE, Little JP. Where does HIT fit? An examination of the affective response to high-intensity intervals in comparison to continuous moderate- and continuous vigorous-intensity exercise in the exercise intensity-affect continuum. PLoS One. 2014; 9(12):e114541; doi: 10.1371/journal.pone.0114541.

41. Malm C, Jakobsson J, Isaksson A. Physical activity and sports - real health benefits: a review with insight into the public health of Sweden. Sports. 2019;7(5):127; doi: 10.3390/sports7050127.

42. Warburton DER, Bredin SSD. Health benefits of physical activity: a systematic review of current systematic reviews. Curr Opin Cardiol. 2017;32(5):541-556; doi: 10.1097/HCO.0000000000000437.

43. Reddy P, Dias I, Holland C, Campbell N, Nagar I, Connolly L, et al. Walking football as sustainable exercise for older adults - a pilot investigation. Eur J Sport Sci. 2017;17(5):638-645; doi: 10.1080/17461391.2017.129 8671.

44. Foulds HJA, Bredin SSD, Charlesworth SA, Ivey AC, Warburton DER. Exercise volume and intensity: a doseresponse relationship with health benefits. Eur J Appl Physiol. 2014;114(8):1563-1571; doi: 10.1007/s00421 -014-2887-9.

45. Randers MB, Nielsen JJ, Krustrup BR, Sundstrup E, Jakobsen MD, Nybo L, et al. Positive performance and health effects of a football training program over 12 weeks can be maintained over a 1-year period with reduced training frequency. Scand J Med Sci Sports. 2010;20(Suppl. 1):80-89; doi: 10.1111/j.1600-0838. 2010.01091.x.

46. Randers MB, Petersen J, Andersen LJ, Krustrup BR, Hornstrup T, Nielsen JJ, et al. Short-term street soccer improves fitness and cardiovascular health status ofhomeless men.EurJ ApplPhysiol.2012;112(6):20972106; doi: 10.1007/s00421-011-2171-1.

47. Gray CM, Hunt K, Mutrie N, Anderson AS, Treweek S, Wyke S. Weight management for overweight and obese 


\section{HUMAN MOVEMENT}

F.M. Clemente et al., Effects of recreational soccer on fat mass

men delivered through professional football clubs: a pilot randomized trial. Int J Behav Nutr Phys Act. 2013;10(1):121; doi: 10.1186/1479-5868-10-121.

48. Krustrup P, Randers MB, Andersen LJ, Jackman SR, Bangsbo J, Hansen PR. Soccer improves fitness and attenuates cardiovascular risk factors in hypertensive men. Med Sci Sports Exerc. 2013;45(3):553-561; doi: 10.1249/MSS.0b013e3182777051.

49. Andersen TR, Schmidt JF, Thomassen M, Hornstrup T, Frandsen U, Randers MB, et al. A preliminary study: effects of football training on glucose control, body composition, and performance in men with type 2 diabetes. Scand J Med Sci Sports. 2014;24(Suppl. 1):43-56; doi: 10.1111/sms.12259.

50. De Sousa MV, Fukui R, Krustrup P, Pereira RMR, Silva PRS, Rodrigues AC, et al. Positive effects of football on fitness, lipid profile, and insulin resistance in Brazilian patients with type 2 diabetes. Scand J Med Sci Sports. 2014;24(Suppl. 1):57-65; doi: 10.1111/ sms.12258.

51. Mohr M, Lindenskov A, Holm PM, Nielsen HP, Mortensen J, Weihe P, et al. Football training improves cardiovascular health profile in sedentary, premenopausal hypertensive women. Scand J Med Sci Sports. 2014; 24(Suppl. 1):36-42; doi: 10.1111/sms.12278.

52. Uth J, Hornstrup T, Schmidt JF, Christensen JF, Frandsen C, Christensen KB, et al. Football training improves lean body mass in men with prostate cancer undergoing androgen deprivation therapy. Scand J Med Sci Sports. 2014;24(Suppl. 1):105-112; doi: 10.1111/ sms.12260.

53. Krustrup P, Skoradal M-B, Randers MB, Weihe P, Uth J, Mortensen J, et al. Broad-spectrum health improvements with one year of soccer training in inactive mildly hypertensive middle-aged women. Scand J Med Sci Sports. 2017;27(12):1893-1901; doi: 10.1111/ sms.12829.

54. Uth J, Hornstrup T, Christensen JF, Christensen KB, Jørgensen NR, Schmidt JF, et al. Efficacy of recreational football on bone health, body composition, and physical functioning in men with prostate cancer undergoing androgen deprivation therapy: 32-week follow-up of the FC prostate randomised controlled trial. Osteoporos Int. 2016;27(4):1507-1518; doi: 10.1007/s00198 -015-3399-0.

55. Hunt K, Wyke S, Gray CM, Anderson AS, Brady A, Bunn C, et al. A gender-sensitised weight loss and healthy living programme for overweight and obese men delivered by Scottish Premier League football clubs (FFIT): a pragmatic randomised controlled trial. Lancet. 2014; 383(9924):1211-1221; doi: 10.1016/S0140-6736(13) 62420-4. 